\title{
A humanização como política pública e suas implicações para a formação em fisioterapia
}

\author{
The humanization as a public policy and its implications \\ for the physiotherapy education
} \author{
Rayanne Mesquita Bendelack ${ }^{4}$ \\ Prefeitura Municipal de Castanhal, Castanhal (PA), Brasil. \\ Universidade do Estado do Pará, Belém (PA), Brasil. \\ ${ }^{3}$ Prefeitura Municipal de São Paulo, São Paulo (SP), Brasil. \\ ${ }^{4}$ Universidade Federal do Pará, Belém (PA), Brasil.
}

Kathlen Oliveira da Silva', Ana Cristina Vidigal Soeiro², Vanessa do Nascimento de Souza ${ }^{3}$

\section{RESUMO}

Introdução: Desde a sua criação, o Sistema Único de Saúde (SUS) conquistou inúmeros e inquestionáveis avanços, entretanto o enfrentamento de diversos desafios no percurso de sua consolidação ainda se faz necessário, incluindo maior integralidade nas práticas em saúde. No cenário atual, com graves problemas nas práticas profissionais, é urgente resgatar a valorização e defesa da vida como eixo norteador da produção de cuidado. Desse modo, o objetivo do estudo foi discutir como a Política Nacional de Humanização da Atenção e da Gestão do SUS (PNH) tem contribuído de modo relevante para a consolidação e qualificação das ações em saúde e para o fortalecimento do trabalho em rede, com destaque para o papel das universidades e instituições formadoras neste cenário de transformações. Trata-se de um estudo exploratório, analítico e descritivo, realizado através de revisão narrativa. Desenvo/vimento: As diretrizes curriculares do ensino em saúde e as crescentes demandas do SUS têm impulsionado mudanças na formação acadêmica e no ensino em saúde. Na Fisioterapia, tendo em vista que a profissão teve seu surgimento e história mais pautados na reabilitação física, a integralidade das ações em saúde e a valorização do compromisso social ainda são grandes desafios na consolidação da humanização como política pública. Considerações Finais: A PNH defende o engajamento de trabalhadores, usuários e gestores, além dos atores sociais que atuam na formação de trabalhadores do SUS como importante estratégia de modificação dos modos de atenção e gestão do trabalho em saúde, priorizando os processos de construção coletiva nas práticas de saúde. Como resultado, é imperativo intensificar a reflexão sobre a humanização enquanto política de saúde e sua importância no enfrentamento dos desafios de fortalecimento do SUS, dentro das universidades e seus diversos cursos, incluindo a Fisioterapia.

PALAVRAS-CHAVE: Humanização. Fisioterapia. Formação profissional em saúde.

Kathlen Oliveira da Silva

Prefeitura do Municipio de Castanhal Av. Barão do Rio Branco, 2232, Centro, CEP 68743-050, Castanhal (PA), Brasil kathlenfisio@gmail.com

\section{FONTE DE FINANCIAMENTO}

Nenhuma.

\section{CONFLITO DE INTERESSE}

Os autores declararam não

O estudo foi realizado na Universidade do Estado do Pará, Belém (PA), Brasil.

Em 2014, recorte deste trabalho foi apresentado sob a modalidade comunicação livre na I Jornada comunicação livre na I Jornada Acadêmica sobre Humanização, Universidade do Estado do Pará, com o título: A Humanização na formação de fisioterapeutas: uma aposta de fortalecimento do Sistema Único de Saúde.

Todos os autores leram e aprovam a versão final submetida ao Interdisciplinary Journal of

Health Education (IJHE).

\section{ABSTRACT}

Introduction: Since its creation, the Unified Health System (SUS) has achieved innumerable and unquestionable advances, however, facing several challenges in the course of its consolidation is still necessary, including greater comprehensiveness in health practices. In the current scenario, with serious problems in professional practices, it is urgent to rescue the valorization and defense of life as the guiding axis of care production. Thus, the objective of the study was to discuss how the National Policy for Humanization of Attention and Management of the Unified Health System has contributed in a relevant way to the consolidation and qualification of health actions and to the strengthening of networking with emphasis on the role of universities and institutions in this scenario of transformations. It is an exploratory, analytical and descriptive study, carried out through a narrative review. Development: The curricular guidelines of health education and the increasing demands of SUS have driven changes in academic training and health education. In Physical Therapy, considering that the profession had its appearance and history more based on physical rehabilitation, the integrality of health actions and the valorization of social commitment are still great challenges in the consolidation of humanization as public policy. Final considerations: The National Policy for Humanization of Attention and Management of the Unified Health System defends the engagement of workers, users and managers as well as the social actors who work in the training of SUS workers as an important strategy for modifying care and management modes of health work, prioritizing the processes of collective construction in health practices. As a result, it is imperative to intensify the reflection on humanization as a health policy and its importance in facing the challenges of strengthening the SUS, within the universities and its various courses, including Physical Therapy.

KEYWORDS: Humanization. Physiotherapy. Health human resource training 
A saúde tem sido concebida através de diversas perspectivas, que continuamente são influenciadas, direta ou indiretamente, pela maneira como as sociedades se organizam e pelas características (históricas, econômicas, políticas e culturais) de cada país ${ }^{1,2}$.

A atenção à saúde no Brasil, tal como está estruturada na atualidade - sistemas de saúde públicos e privados - tem uma construção recente, deflagrada em meados do século passado, sendo o surgimento do Sistema Único de Saúde (SUS), um marco histórico importante neste processo ${ }^{2,3}$.

O SUS teve sua criação formal na Constituição Federal de 1988, mas vem se estruturando há muito mais tempo. Os fatores que contribuíram para o seu surgimento - por exemplo, a crise do modelo médico assistencial privatista, as mudanças políticas e econômicas dos anos 1970 e 1980 e o processo de redemocratização do país - determinaram novos rumos nas políticas públicas e nos sujeitos sociais, que passaram a lutar por um modelo alternativo de atenção à saúde ${ }^{4}$.

Os inúmeros avanços do SUS são inquestionáveis, mas ainda persistem problemas de diversas ordens a serem enfrentados, os quais incluem: a fragmentação e a verticalização dos processos de trabalho, que limitam as relações entre os diferentes profissionais da saúde e entre estes e os usuários; os modos de atenção, baseados na relação queixa-conduta, e o modelo de formação dos profissionais de saúde, que se mantém distante do debate e da formulação das políticas públicas de saúde ${ }^{5}$. Todos esses fatores têm sido apontados como desafios do SUS, os quais demandam mudanças estruturais na forma como está organizada a saúde no território brasileiro.

Em meio aos debates sobre os desafios do SUS, a partir da primeira década do século XXI, o termo humanização começa a ganhar maior espaço na literatura de saúde, assumindo diferentes significados ${ }^{6}$, em especial por seu caráter polissêmico ${ }^{7-9}$ e multidimensional. Tal fato reflete na falta de clareza e incompreensão presentes no imaginário popular no que tange ao uso da expressão, ainda que ela tenha sido repetidamente usada como alusão a práticas desejáveis no SUS.

A partir do ano 2000, a humanização teve destaque crescente no cenário histórico da saúde no Brasil ${ }^{10}$, porém na forma de um discurso ainda distanciado das mudanças concretas nas práticas de saúde 7 . É nesse contexto, que muitos projetos de humanização se desenvolveram em áreas específicas da assistência, como a humanização do parto, a humanização da assistência à criança, o Projeto Maternidade Segura e o Método Cangurún ${ }^{11}$.

No ano de 2001, o Ministério da Saúde (MS) criou o Programa Nacional de Humanização da Atenção Hospitalar (PNHAH), o qual oferecia uma diretriz global que agrupava os projetos de humanização já desenvolvidos nas diversas áreas de atendimento hospitalar e tinha por objetivo fundamental enfrentar os grandes desafios de melhoria da qualidade do atendimento público à saúde e de valorização do trabalho dos profissionais desta área, bem como aprimorar as relações interpessoais (relações entre profissionais, entre profissionais e usuários e, por fim, entre a comunidade e o hospital) pela compreensão dos fenômenos no campo das subjetividades ${ }^{12,13}$.

O programa surgiu como reflexo das condições de saúde em evidência naquele momento e por influência da $11^{\text {a }}$ Conferência Nacional de Saúde, que anunciava de maneira tímida a abordagem sobre a humanização da atenção à saúde em seu título ${ }^{14 *}$.

Neste ínterim, o cenário era ambíguo: usuários e alguns trabalhadores reivindicavam humanização e a maioria dos gestores e dos profissionais a secundarizava, ou mesmo a banalizava? .

Apesar da importância de tais iniciativas para o desenvolvimento de uma política de humanização, Benevides e Passos ${ }^{7}$ ressaltam que todas estas medidas ainda expressavam uma baixa horizontalidade e predomínio da verticalização no modo

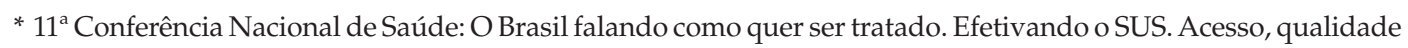
e humanização da atenção à saúde com controle social.
} 
como tais programas estavam organizados dentro do MS e do SUS. Tais evidências chamaram a atenção para a urgência de se encontrar outras respostas para, o que no dizer de muitos, se tratava da falência do modelo SUS. Segundo os autores ${ }^{7}$, os discursos apontavam para a necessidade de reconstrução do conceito de humanização e de criação de uma política pública e transversal de humanização da/na saúde.

No início de 2003, no MS, intensificou-se o debate em torno das condições precárias de trabalho, das dificuldades de pactuação das diferentes esferas do SUS, do descuido e da falta de compromisso na assistência ao usuário dos serviços de saúde ${ }^{7}$. Esta discussão culminou com o lançamento, neste mesmo ano, da Política Nacional de Humanização da Atenção e Gestão do SUS (PNH), em uma tentativa de enfrentamento para os problemas existentes na atenção e gestão em saúde ${ }^{14}$.

A PNH, também conhecida como HumanizaSUS, é uma política do SUS ${ }^{8}$, que retoma os ideais da reforma sanitária, o movimento constituinte, no sentido de rever e problematizar os modos de fazer no campo das práticas de saúde presentes no cotidiano dos serviços, com abrangência em diferentes territórios ${ }^{15}$. A PNH nasce reconhecendo que há um SUS que dá certo e, embora admita que existam desafios e problemas, aposta na capacidade de superá-los com os próprios acúmulos do SUS ${ }^{10,14}$.

Humanizar, sob a perspectiva da PNH, significa valorizar os diferentes sujeitos (trabalhadores, gestores e usuários) implicados no processo de produção de saúde, através do estímulo à autonomia, protagonismo e corresponsabilidade, o que implica no fomento à participação coletiva nas práticas de saúde ${ }^{16,17}$.

\section{Por que uma política pública para humanizar o SUS?}

Importantes desafios ainda precisam ser superados para que aconteça a consolidação do SUS como política efetiva de universalização do direito à saúde ${ }^{4}$. Considerar o SUS como uma obra aberta é essencial para esse processo de enfrentamento dos desafios atuais, reativando sua força constituinte. As transformações das práticas em saúde "[...] são pensadas como o desafio central no debate das políticas de saúde e a base primordial para a sustentabilidade e legitimidade do SUS na atualidade"18:1.

A PNH é compreendida, nas palavras de Pasche e Passos ${ }^{14: 4}$, como "[...] um processo de criação que a um só tempo envolve dimensões éticas, estéticas e política [...]". Ética, porque implica em mudança de atitudes, comprometimento e co-responsabilidade dos usuários, dos gestores e trabalhadores de saúde em relação à qualidade das ações e serviços gerados. Estética, por envolver processo de produção/criação da saúde e de subjetividades autônomas e protagonistas. E política, porque tem relação com a organização social e institucional das práticas de atenção e gestão na rede do SUS ${ }^{15}$.

Neste contexto, Navarro e Pena ${ }^{17}$ alertam para a necessidade de concretizar o deslocamento da humanização das práticas para uma Política de Humanização do SUS, que além de princípios e método, propõe diretrizes e dispositivos como ofertas para o processo de humanização. Os dispositivos podem ser arranjos materiais e/ou imateriais, que fazem funcionar ou potencializam um processo ${ }^{14}$. "Um dispositivo é uma ação, um projeto, uma tecnologia a ser implementada, algo que dispare um movimento de mudança para transformar as práticas vigentes, tanto na atenção quanto na gestão em saúde"16:22. São exemplos de dispositivos: Acolhimento com Classificação de Risco; Colegiados Gestores; Programa de Formação em Saúde e Trabalho; Equipes de Referência e de Apoio matricial; Projetos de Ambiência; Direito de Acompanhante e Visita Aberta e Construção de Processos Coletivos ${ }^{14}$.

Entendendo que os princípios, o método, as diretrizes e os dispositivos apontam para a necessidade de mudanças no processo de gestão e atenção em saúde, Pasche e Passos ${ }^{14}$ destacam que a PNH surge com a tarefa desafiadora de produzir novas atividades por parte dos sujeitos envolvidos nesse processo. Segundo esses autores, a aproximação entre os sujeitos, na produção de atividades em saúde, fortalece o respeito 
à vida e os direitos dos cidadãos, o estreitamento de vínculos entre trabalhadores, gestores e usuários, a consolidação do trabalho em equipe multiprofissional, a ampliação das redes de produção de saúde, o compromisso com a democratização das relações e a valorização dos profissionais de saúde.

Trata-se de uma política construída através de experiências concretas do "SUS que dá certo"*** , que devem ser aprimoradas e multiplicadas ${ }^{16}$. Os projetos de humanização devem nortear as ações nas diversas áreas e instituições para favorecer as melhorias na qualidade de trabalho e saúde ${ }^{11}$.

A humanização como política é um incentivo a novas práticas de gerir a saúde, repercutindo na concretização/criação de novos modos de fazer ${ }^{17}$, com intuito de ampliar a capacidade do SUS como agente multiplicador de saúde ${ }^{19}$. "Enfim, pensar a humanização enquanto política significa menos o que fazer e mais como fazer"13:5.

Para Heckert e Neves ${ }^{20}$, os processos de produção de saúde configuram-se em uma rede complexa de relações entre sujeitos. E assim como os demais desafios da concretização do SUS, tais processos devem ser debatidos durante a formação dos trabalhadores de saúde, produzindo interferência nos modos instituídos de gestão e de produção de cuidado.

\section{Formação de sujeitos implicados no/para o SUS: a humanização na formação do fisioterapeuta}

Sabe-se que a rede de serviços do SUS é, por excelência, espaço de formação na área da saúde ${ }^{21}$ e por isso defende-se que os processos de formação sejam peça primordial para que futuros profissionais estejam inseridos na consolidação do SUS de forma efetiva ${ }^{10}$.

As Instituições de Ensino Superior (IES) em saúde tem essencial influência no direcionamento desta formação, e devem estimular, dentre outros projetos, a interação do processo de ensino-pesquisa-extensão nos novos cenários ${ }^{22}$, por permitir ao estudante contextualizar melhor seu papel social através de contribuições concretas às redes prestadoras de serviço ao longo de toda a graduação.

Para isso, é necessário que tais instituições rompam com o modelo convencional e consolidem as novas Diretrizes Curriculares Nacionais (DCN), estando sempre abertas às novas metodologias de ensino e às novas políticas de saúde disparadas pelo Estado, a exemplo da PNH.

A humanização, timidamente comentada no texto das DCN, surge como aposta importante para os processos de formação, os quais também são pautas das discussões problematizadas pela $\mathrm{PNH}$, que em um de seus textos básicos preconiza que a humanização componha o conteúdo profissional da graduação, pós-graduação e da extensão em saúde, vinculando-a aos Polos de Educação Permanente e às instituições formadoras ${ }^{10,15}$.

Ao tratar de processos de formação, a PNH não se baseia em uma concepção abstrata, mas em experimentação desses processos, especialmente a partir de experiências concretas de formação de apoiadores do HumanizaSUS, a qual é uma estratégia para exercitar as diretrizes e o método da PNH e para capilarizá-la como política do SUS ${ }^{16}$.

A PNH, invariavelmente, reflete a necessidade de reorganização dos serviços de saúde e, dessa forma, de restruturação pedagógica dos cursos da área da saúde. Nesse aspecto, a interlocução com as instituições de formação configura-se como um desafio, dentre os vários outros, no processo de humanização do SUS, tendo como finalidade a intervenção na construção dos modelos curriculares de graduação e pós-graduação

** Expressão muito usada na literatura referente à PNH para se referir às experiências exitosas na rede SUS. 
em saúde, de modo a ampliar a visão dos estudantes acerca dos modelos de gestão e atenção ${ }^{23}$.

A luta pela democratização institucional das práticas de saúde, para além dos trabalhadores, gestores e usuários, requer a inclusão de outros atores sociais, como os docentes, os preceptores, a equipe técnica que atua na formação de trabalhadores do SUS, priorizando os processos de construção coletiva. Pois, segundo Casate ${ }^{3: 25}$, é necessário "[...] transformar a formação para possibilitar a construção do cuidado humanizado [...]", e isso sugere revisão, nos modos de relacionamento professoraluno, nos conteúdos e métodos de ensino, na formação de professores e, inclusive, no próprio papel social da universidade ${ }^{10}$.

O panorama de disputa entre o modelo médico-assistencial privatista e o modelo sanitarista ultrapassa o plano da organização dos serviços de saúde e revela-se também no campo da educação/formação profissional em saúde e, no Brasil, tal embate se intensificou a partir da criação do SUS, que colocou em destaque a necessidade de um novo perfil profissional ${ }^{21,24}$.

Nesse sentido, as DCN surgem para estimular a inserção precoce e progressiva do acadêmico no SUS, garantindo-lhe conhecimento e compromisso com a realidade de saúde da sua região e do seu país. Além disso, renovam o já disposto na Lei Orgânica da Saúde, que diz respeito à ação interdisciplinar, de modo a alcançar a integralidade de assistência ao indivíduo e à coletividade ${ }^{25}$.

Os valores sociais vigentes, o modelo econômico e político e a forma de organização dos serviços de saúde influenciam diretamente a formação e o perfil dos profissionais da área ${ }^{1}$. Além disso, as modificações na realidade demográfica e epidemiológica de cada país alteram as necessidades e demandas populacionais por atenção à saúde, o que traz importantes consequências para a educação profissional em saúde. Tal constatação reflete a urgente necessidade de articulação entre a formação profissional e a organização do sistema de saúde 22,24 .

O desenvolvimento precoce do trabalho em equipe e a inserção do aluno em atividades que imprimam crescimento gradativo de responsabilidade e reforcem a compreensão da importância de cada profissional contribuem para a construção da integralidade na atenção à saúde ${ }^{22}$. Nesse aspecto, a vivência de discentes e docentes, os debates entre os profissionais e acadêmicos nos diferentes níveis de atenção do SUS deve ser iniciada ainda nas etapas de formação profissional, sempre visando superar os desafios enunciados pela sociedade brasileira quanto à qualidade e à dignidade no cuidado em saúde ${ }^{10}$.

A assistência prestada à comunidade reflete a necessidade de estudar e trabalhar a saúde em sua totalidade social ${ }^{26}$. Essas atitudes permitem enxergar o sujeito na sua complexidade, a partir da compreensão do seu contexto para além das expressões da doença em si, fato que implica na ampliação da clínica com uma abordagem interdisciplinar e multiprofissional no cuidado do indivíduo ${ }^{15}$.

Infelizmente, mesmo após tantas transformações, a formação acadêmica dos profissionais de saúde ainda está pautada, majoritariamente, na prática curativa desenvolvida em serviços de atenção secundária e terciária, em que predomina a atuação focada na doença, reduzindo o sujeito ao território biológico ${ }^{1}$.

Nesse contexto de mudanças, pode-se afirmar que formar profissionais para o SUS não é tarefa tão fácil, mas parece ser ainda mais difícil para as profissões que tiveram na sua origem, atuação apenas na assistência "reabilitadora", no tratamento de sequelas, a exemplo da fisioterapia ${ }^{1,27}$.

Os níveis secundário e terciário (centros de reabilitação e hospitais) de atenção à saúde são os locais de maior atuação do profissional fisioterapeuta ${ }^{1}$, fato que, teoricamente, o exime de grande responsabilidade social ${ }^{26}$. E basta retomar a história da Fisioterapia como ciência e como profissão para perceber que sua origem guarda íntima relação com um modelo mais tecnicista e distante das disciplinas do campo social. 
O ensino superior de fisioterapia, aparentemente, ainda na atualidade, volta-se ao ensino de técnicas e moldes de atuação profissional ao invés de focar-se no desenvolvimento de sua atuação de maneira socialmente significativa. Assim, introduzir a profissão, com o enfoque relevante das Políticas Públicas de Saúde, é uma necessidade fundamental na busca da consolidação da saúde e de uma visão mais crítica da realidade para esses profissionais ${ }^{27}$.

A fisioterapia, como uma profissão da saúde, está habilitada a desenvolver serviços de prevenção de doenças, promoção e recuperação da saúde ${ }^{26}$, podendo agregar funções importantes na assistência à comunidade, que comumente tem acesso restrito a esses serviços, devido à concentração dos profissionais no atendimento privado, estimulada até mesmo pela sua formação direcionada para especialidades, a exemplo da fisioterapia dermato-funcional (estética), reeducação postural global, pilates e acupuntura, frente aos desejos e necessidades dos usuários-pagantes ${ }^{1}$.

Como profissional de saúde, o profissional fisioterapeuta pode atuar em todos os níveis de atenção à saúde e possui importante potencial para desenvolver atividades no campo da Saúde Coletiva, integrando-se à equipe de saúde, alcançando a coletividade e intervindo positivamente na melhoria das condições de vida desse grupo de pessoas ${ }^{26}$.

Entretanto, o estabelecimento da profissão nesse nível de atenção, requer trabalho árduo e mudanças graduais de indivíduo a indivíduo, até o momento em que esse campo faça parte do cotidiano profissional de toda a categoria, desde a formação ${ }^{26}$, para que os profissionais estejam bem preparados e informados sobre as políticas de saúde e particularmente sobre o SUS, o que implica em capacitação do profissional para a ação preventiva e educativa, de modo a assistir a comunidade em que atua, contribuindo para a melhora da qualidade de vida dessa população ${ }^{21}$.

Vivenciar outras formas de atuação que não se restrinjam à tradicional atuação da fisioterapia na reabilitação viabiliza a ampliação das possibilidades do fisioterapeuta, sem, contudo, desvalorizar o papel reabilitador no qual a fisioterapia foi originada e se desenvolveu ${ }^{28}$.

Infelizmente, ainda há poucos profissionais com essa disposição, como afirma Bispo $^{1}$, assinalando que há um grande contingente de profissionais fisioterapeutas aptos a prestar assistência, mas uma população desassistida e com carência de oferta de serviços de saúde, o que demonstra um paradoxo no campo de atuação profissional.

O fisioterapeuta, assim como os demais profissionais de saúde, pode assumir um papel fundamental na construção de novas formas de organização dos serviços de saúde. Contudo, apesar desse potencial, tem-se discutido que a fisioterapia ainda desempenha um papel secundário nesse processo, fato que pode ser explicado, entre outros fatores, justamente pela formação universitária que atribui pouca importância às discussões sobre temas sociais e análise da realidade e, pela prática profissional voltada prioritariamente para apenas uma parte de seu objeto de trabalho, que é a reabilitação $0^{1,26,27}$.

Os tempos atuais exigem que o fisioterapeuta seja capaz de atuar na integralidade da atenção à saúde, imprimindo em seu trabalho uma atitude mais humanizada e trabalhando em equipe multiprofissional, através da efetiva integração com outros profissionais e setores da área da saúde. Essas características são indispensáveis ao profissional que irá atuar em serviços do SUS ${ }^{26}$.

Ampliar o debate sobre a humanização e sua relação com a formação para o trabalho em saúde requer a criação e ampliação de espaços de discussão nas universidades e centros de pesquisa na área da saúde e este tema ainda costuma ser pouco abordado na Fisioterapia ${ }^{28}$. Trata-se de um modo de reposicionar a formação no SUS e para o SUS ${ }^{19}$ e de intervir nas práticas cotidianas dos serviços e na promoção e potencialização da saúde, conforme os princípios estabelecidos pelo SUS.

A PNH defende a inseparabilidade entre "formação-intervenção". Deste modo, a formação deve ser um exercício de experimentação do convívio, de troca entre sujeitos 
em situações reais e concretas do cotidiano dos serviços de saúde e é a qualidade e intensidade desta troca que possibilita bons processos de formação 9,19.

Diante deste cenário, é necessário que as universidades e seus cursos, incluindo neste cenário a Fisioterapia, formem cidadãos-trabalhadores da saúde com capacidade técnica e política para construir novas realidades institucionais e novas práticas, mais eficazes, mais justas e igualitárias.

Para alguns autores ${ }^{29}$, a instituição das vivências defendidas pela PNH no âmbito acadêmico-profissional pode contribuir gradativamente à assimilação desse processo. Entretanto, "[...] embora a PNH tenha como um de seus eixos de implementação a inserção das diretrizes da humanização nas escolas formadoras de profissionais da área da saúde, na prática, sua presença no ensino superior ainda é pálida e sôfrega"13:7.

As reflexões sobre os temas aqui abordados vêm ganhando espaço nos serviços de saúde e instituições de ensino do Brasil ${ }^{8}$. Contudo, para a consolidação desses ideais nas práticas em saúde, é necessário que a Política de Humanização esteja instituída nas práticas do gerir saúde e nos mecanismos de produção de praticantes do cuidado ${ }^{19}$. Transcorridos mais de dez anos desde a criação da PNH, ainda há um longo caminho para que as universidades potencializem nas suas salas de aula esta discussão, superando inclusive o desconhecimento ${ }^{28}$ de sua dimensão como política pública $^{10}$.

Fomentar a difusão da PNH de forma transversal nas instituições formadoras é apostar, dentre outras coisas, em um reencantamento no SUS capaz de resgatar os ideais reformistas naqueles que provavelmente não viveram os anos de luta democrática, mas que possuem igualmente potencial para mudar as práticas de saúde no Brasil. É inegável que as diferenças entre a região norte e as demais regiões do país apontam para a necessidade de considerar as peculiaridades de cada cenário, mas sem desconsiderar que o SUS é, sobretudo, um patrimônio de toda a sociedade brasileira.

\section{Referências}

1. Bispo JP Jr. Formação em fisioterapia no Brasil: reflexões sobre a expansão do ensino e os modelos de formação. Hist Cienc Saude Manguinhos. 2009;16(3):655-68. http://dx.doi.org/10.1590/S0104-59702009000300005. PMid:20614669.

2. Santos JGW. Os impactos da gestão dos processos de trabalho sobre a atividade de profissionais de saúde de um hospital universitário da região metropolitana de Belém-PA [tese]. Belém (PA): Universidade Federal do Pará; 2011. 223 p.

3. Casate JC. A humanização do cuidado na formação dos profissionais de saúde nos cursos de graduação. Ribeirão Preto [dissertação]. Ribeirão Preto (SP): Escola de Enfermagem de Ribeirão Preto, Universidade de São Paulo; 2010.31 p.

4. Brasil. Conselho Nacional de Secretários de Saúde. SUS: Avanços e Desafios. Brasília (DF): CONASS; 2006 [citado em 2017 Jul 18]. 166 p. Disponível em: https://edisciplinas.usp.br/pluginfile.php/2554917/mod_resource/content/0/susavancosedesafios. pdf

5. Brasil.Ministério da Saúde. HumanizaSUS: Política Nacional de Humanização, a humanização como eixo norteador das práticas de atenção e gestão em todas as instâncias do SUS. Brasília (DF): MS; 2004 [citado em 2017 Jul 18]. 20 p. Disponível em: http://bvsms.saude.gov.br/bvs/publicacoes/humanizasus_2004.pdf

6. Casate JC, Corrêa AK. A humanização do cuidado na formação dos profissionais de saúde nos cursos de graduação. Rev Esc Enferm USP. 2012;46(1):219-26. http://dx.doi.org/10.1590/S0080-62342012000100029. PMid:22441287.

7. Benevides R, Passos E. A humanização como dimensão pública das políticas de saúde. Cien Saude Colet. 2005;10(3):56171. http://dx.doi.org/10.1590/S1413-81232005000300014.

8. Barbosa GC, Meneguim S, Lima SAM, Moreno V. Política Nacional de Humanização e formação dos profissionais de saúde: revisão integrativa. Rev Bras Enferm. 2013;66(1):123-7. http://dx.doi.org/10.1590/S0034-71672013000100019. PMid:23681390.

9. Medeiros LOP, Batista SHSS. Humanização na formação e no trabalho em saúde: uma análise da literatura. Trab Educ Saúde. 2016;14(3):925-51. http://dx.doi.org/10.1590/1981-7746-sol00022.

10. Moreira MADM, Lustosa AM, Dutra F, Barros EO, Batista JBV, Duarte MCS. Políticas públicas de humanização: revisão integrativa da literatura. Cien Saude Colet. 2015;20(10):3231-42. http://dx.doi.org/10.1590/1413-812320152010.10462014. PMid:26465863.

11. Mota RA, Martins CGM, Véras RM. Papel dos Profissionais de Saúde na Política de Humanização Hospitalar. Psicol Estud. 2006;11(2):323-30. http://dx.doi.org/10.1590/S1413-73722006000200011.

12. Brasil. Ministério da Saúde. Programa Nacional de Humanização da Atenção Hospitalar. Brasília (DF): MS; 2001 [citado em 2017 Jul 18]. 60 p. Disponível em: http://bvsms.saude.gov.br/bvs/publicacoes/pnhah01.pdf

13. Rios IC. Humanização: a essência da ação técnica e ética nas práticas de saúde. Rev Bras Educ Med. 2008;33(2):253-61. http://dx.doi.org/10.1590/S0100-55022009000200013. 


\section{ijhe}

14. Pasche DF, Passos E. A importância da humanização a partir do Sistema Único de Saúde. Rev Saúde Púb de Sta Catarina [Internet]. 2008 Jan-Jun [citado em 2017 Jul 18];1(1):92-100. Disponível em: http://revista.saude.sc.gov.br/index.php/inicio/ article/view/19/82

15. Brasil. Ministério da Saúde. Política Nacional de Humanização [Internet]. Brasília (DF): MS; 2004 [citado em 2017 Jul 18 ]. 32 p. Disponível em: http://bvsms.saude.gov.br/bvs/publicacoes/humanizaSUS_politica_nacional_humanizacao.pdf

16. Brasil. Ministério da Saúde. Secretaria de Atenção à Saúde. Política Nacional de Humanização. Redes de Produção de Saúde. Brasília (DF): MS; 2009 [citado em 2017 Jul 18]. 20 p. Disponível em: http://bvsms.saude.gov.br/bvs/publicacoes/ redes_producao_saude.pdf

17. Navarro LM, Pena RS. A Política Nacional de Humanização como estratégia de produção coletiva das práticas em saúde. Rev de Psicol da UNESP. [Internet]. 2013 Jun [citado em 2017 Jul 18];12(1):64-73. Disponível em: http://pepsic.bvsalud. org/scielo.php?script=sci_arttext\&pid=S1984-90442013000100007\&lng=pt\&nrm=iso\&tlng=pt

18. Neves CAB. Que vida queremos afirmar na construção de uma política de humanização nas práticas de saúde do Sistema Único de Saúde (SUS)? Interface: Comunic Saúde Educ. [Internet]. 2009 [citado em 2017 Jul 18];13(1):781-95. Disponível em: http://www.redalyc.org/pdf/1801/180115446029.pdf

19. Pasche DF. Política Nacional de Humanização como aposta na produção coletiva de mudanças nos modos de gerir e cuidar. Interface: Comunic, Saúde Educ. 2009;13(1):701-8. http://dx.doi.org/10.1590/S1414-32832009000500021.

20. Heckert ALC, Neves CAB. Modos de formar e modos de intervir: quando a formação se faz potência de produção de coletivo. In: BRASIL. Ministério da Saúde. Cadernos HumanizaSUS: formação e intervenção. Brasília (DF): MS; 2010 [citado em 2017 Jul 18]. p. 13-27. Disponível em: http://bvsms.saude.gov.br/bvs/publicacoes/cadernos_humanizaSUS.pdf

21. Silva DJ, Ros MA. Inserção de profissionais de fisioterapia na equipe de saúde da família e Sistema Único de Saúde: desafios na formação. Cien Saude Colet. 2007;12(6):1673-81. http://dx.doi.org/10.1590/S1413-81232007000600028. PMid:18813503.

22. Motta LB, Aguiar AC. Novas competências profissionais em saúde e o envelhecimento populacional brasileiro: integralidade, interdisciplinaridade e intersetorialidade. Cien Saude Colet. 2007;12(2):363-72. http://dx.doi.org/10.1590/S141381232007000200012. PMid:17680091.

23. Heckert ALC, Passos E, Barros MEB. Um seminário dispositivo: a humanização do Sistema Único de Saúde (SUS) em Debate. Interface: Comunic Saúde e Educ. 2009;13(1, suppl 1):493-502. http://dx.doi.org/10.1590/S1414-32832009000500002.

24. Haddad AE, Morita MC, Pierantoni CR, Brenelli SL, Passarella T, Campos FE. Formação de profissionais de saúde no Brasil. Rev Saude Publica. 2010;44(3):383-93. http://dx.doi.org/10.1590/S0034-89102010005000015. PMid:20499011.

25. Brasil. Ministério da Educação. Conselho Nacional de Educação. Parecer CNE/CES 1.210/2001. Diretrizes Curriculares Nacionais dos Cursos de Graduação em Fisioterapia, Fonoaudiologia e Terapia Ocupacional. Diário Oficial da República Federativa do Brasil. Brasília (DF): MEC; 2001 [citado em 2017 Jul 18]. Disponível em: http://portal.mec.gov.br/cne/ arquivos/pdf/pces1210_01.pdf

26. Salmória JG, Camargo WA. Uma aproximação dos signos - fisioterapia e saúde - aos aspectos humanos e sociais. Saúde Soc São Paulo. 2008;17(1):73-84. http://dx.doi.org/10.1590/S0104-12902008000100007.

27. Jesus HJA. O Fisioterapeuta no SUS: contribuição para as políticas públicas de saúde em Belém/PA. Fortaleza [dissertação]. Itaperi: Universidade Estadual do Ceará; 2010. 164 p.

28. Silva ID, Silveira MFA. A humanização e a formação do profissional em fisioterapia. Cien Saude Colet. 2011;16(1, suppl 1):1535-46. http://dx.doi.org/10.1590/S1413-81232011000700089. PMid:21180829.

29. Sumiya A, Jeolás LS. Processos de mudança na formação do fisioterapeuta: as transições curriculares e seus desafios. Acta Sci Hum Soc Sci. 2010;32(1):47-53.

\section{Contribuição dos autores}

Kathlen Oliveira da Silva participou da concepção inicial, confecção e revisão do manuscrito; Ana Cristina Vidigal Soeiro participou como orientadora na concepção inicial, elaboração e revisão do manuscrito; Vanessa do Nascimento de Souza e Rayanne Mesquita Bendelack participaram da elaboração e revisão do manuscrito. 